\title{
Seizures in Pregnancy- A Rare Case of Neurocysticercosis Mimicking Eclampsia
}

\author{
Pooja Chandrakant Kshirsagar ${ }^{1}$, Neema Acharya ${ }^{2}$, Sourya Acharya ${ }^{3}$, Amardeep Tembhare ${ }^{4}$
}

\begin{abstract}
${ }^{1}$ Department of Obstetrics and Gynaecology, Datta Meghe Institute of Medical Sciences, Wardha, Maharashtra, India. ${ }^{2}$ Department of Obstetrics and Gynaecology, Datta Meghe Institute of Medical Sciences, Wardha, Maharashtra, India. ${ }^{3}$ Department of Medicine, Datta Meghe Institute of Medical Sciences, Wardha, Maharashtra, India.

${ }^{4}$ Department of Obstetrics and Gynaecology, Datta Meghe Institute of Medical Sciences, Wardha, Maharashtra, India.
\end{abstract}

\section{INTRODUCTION}

Seizures in pregnancy are commonly due to eclampsia if it occurs after 20 weeks of gestation, unless proved otherwise. Other causes are epilepsy and rarely any disorder related to central nervous system. In pregnancy, convulsions along with atypical clinical features can lead to other differential diagnosis. Though Neurocysticercosis is rare, it is still a significant cause of convulsions in pregnancy. Diagnosis is made on the basis of clinical presentation and is confirmed by radiological features and immunological testing. Medical Management includes antiepileptics and antiparasitic agents. We report a case of seizures in a known case of preeclamptic primigravida with 34 weeks of gestation mimicking eclampsia who had neurocysticercosis brain as aetiology of seizure.

Eclampsia is the most common cause of convulsions after 20 weeks of pregnancy. Rarely patient may have other co-existing causes such as epilepsy, tuberculoma, neurocysticercosis, and space occupying lesions which may be missed in cases of preeclampsia leading to delayed diagnosis and management. Neurocysticercosis (NCC), although rare, may lead to convulsions in pregnancy.[1] It is caused by the larvae of Taenia solium which infects the central nervous system (CNS). This condition is called as cysticercosis or, when the central nervous system is involved, it is known as neurocysticercosis.[2] Brain parenchyma is the commonest site involved and the most common symptom is seizures. Clinical features ranges from a single seizure to coma and death. Medical treatment is effective in most of the cases although surgery may be indicated in a few patients. [3] We report a case of seizures in a known case of preeclamptic primigravida with 34 weeks of gestation mimicking eclampsia who had neurocysticercosis brain as aetiology of seizure.

\section{PRESENTATION OF CASE}

A 34 years old primigravida presented to the casualty with 28 weeks of gestation with severe headache and 4 episodes of vomiting followed by 2 episodes of generalized tonic-clonic convulsions. There was no history of any chronic illness (diabetesmellitus, chronic-hypertension, bronchial-asthma, thyroid disorder, tuberculosis). Patient was diagnosed as pre-eclampsia at 26 weeks for which she was taking Tab labetalol $100 \mathrm{mg}$ twice daily doses. Diagnosis of eclampsia was made. On examination, she was conscious, alert and had a blood pressure of 140/90 $\mathrm{mmHg}$, with bilateral pitting type pedal oedema, there was +1 albuminuria. Fundoscopy, renal and liver function tests were normal. Treatment for eclampsia was started with Sibai regimen \& anti hypertensives. Patient also gave history of mild to moderate headache during last 1 week which got aggravated in the last 6 hours and was associated with diplopia followed by convulsion. In view of this presentation MRI brain was done which revealed 2 small rounded hyperintensities noted on T2 weighted involving parieto-occipital region on both sides (Figure 1).
Corresponding Author: Dr. Neema Acharya, Professor, Department of Obstetrics and Gynaecology, Datta Meghe Institute of Medical Sciences, Wardha, Maharashtra, India.

E-mail: neemasacharya@gmail.com

DOI: $10.14260 / j e m d s / 2020 / 217$

Financial or Other Competing Interests: None.

How to Cite This Article:

Kshirsagar PC, Acharya N, Acharya S, et al. Seizures in pregnancy- a rare case of neurocysticercosis mimicking eclampsia. J. Evolution Med. Dent. Sci. 2020;9(12): 10121014, DOI: 10.14260/jemds/2020/217

Submission 08-01-2020,

Peer Review 27-02-2020,

Acceptance 05-03-2020,

Published 23-03-2020.

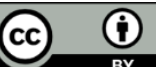


There was no E/O of hyperintensities and oedema in other regions of brain like those seen in preeclampsia. A diagnosis of neurocysticercosis was made on the basis of the MRI. She was put on levetiracetam by the consultant neurologist for the same. Antihelminthics were not started as risk of inflammatory response was present. Being from very remote area and having precious pregnancy along with two high risk conditions she was managed on indoor basis. The symptoms resolved in 1 week and pregnancy could be conserved for next 6 weeks with the multidisciplinary management by an obstetrician, neurologist and physician. Patient had mild preeclampsia well controlled with labetalol, but caesarean section had to be done for mild FGR (foetal growth restriction) with abnormal obstetric Doppler findings (absent diastolic flow in umbilical artery) at 34 weeks of gestation age. Her intra operative immediate postoperative period was uneventful and she was discharged with a healthy male baby of $2 \mathrm{~kg}$.

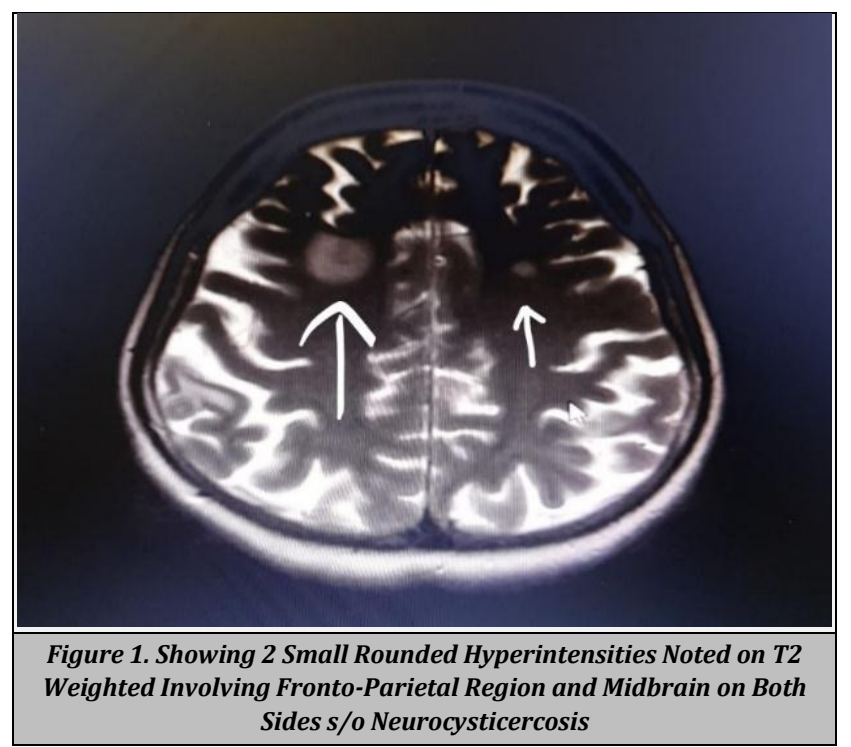

DISCUSSION

This case was initially diagnosed as eclampsia due to preexisting preeclampsia. The other neurological symptoms were not typical of mild preeclampsia. Bearing this in the mind, an emergency MRI of the head was done which detected the spherical ring-enhancing lesion in both parieto-occipital lobes as seen in figure. The diagnosis of neurocysticercosis was made on the basis of MRI findings, symptoms and living in an endemic area. This case is unique as patient presented with convulsions in a known case of pre-eclampsia and the diagnosis of neurocysticercosis would have been missed and we may have planned an emergency termination for such a precious pregnancy. In this case diplopia was a symptom which led us to investigate and consult a neurologist and do MRI.

Many neurologic disorders may complicate pregnancy and affect the treatment of an otherwise normal obstetric case.[4] In pregnancy the most common cause of convulsions is eclampsia. Eclampsia is defined as seizures or coma of a pregnant woman, with signs and symptoms of preeclampsia, and no other cerebral conditions. However, Sibai and Fair lie.[5] reviewed 200 patients with eclampsia and documented that
$20 \%$ had blood pressure lower than $140 / 90 \mathrm{~mm} \mathrm{Hg}$ and $22 \%$ were without proteinuria. If the eclampsia is not controlled on medications and any other atypical features are present then we have to rule out other causes of convulsions in pregnancy by neuroimaging. Del-Brutto has defines some definitive and probable criteria for the diagnosis of neurocysticercosis: histology; radiological imaging; epidemiology; serology; clinical features; and follow-up scans.[1] In pregnancy the same criteria is applied for the diagnosis of NCC as for the general population.[6] Magnetic response imaging (MRI) is superior to a computed tomography (CT) scan in diagnosis and follow-up studies.[2] The diagnosis of NCC can be done by MRI (as MRI is preferred over the CT SCAN in pregnancy).Also the Enzymelinked Immune-Electro-Transfer blot (EITB) assay detects serum antibodies to Taenia Solium with the sensitivity of $98 \%$ in cases with more than one parenchymal cyst or subarachnoid disease. If EITB is not available, CSF can be tested using ELISA to detect anti-cysticercal antibodies.[7]

In general population, the most common presentation of NCC includes headaches and seizures.[8] During pregnancy, headache along with raised intracranial pressure and focal and generalized seizures seem to be more common. ${ }^{[9]}$ Altered mental status, coma, and maternal mortality have also been reported.[10,11,12] The new onset of seizure associated with NCC can be confused for pre-eclampsia and eclampsia particularly after the first trimester of pregnancy.[11],[13,14]

In most of the cases the infection is asymptomatic as has been demonstrated in population-based studies where neurocysticercosis is endemic. ${ }^{[15]}$ Among the clinical features, seizures are the most common form of presentation occurring in up to $80 \%$ of symptomatic infections ${ }^{[16]}$ other features include focal neurological signs, headache. In patients with NCC headache, nausea, and vomiting (symptoms of hydrocephalus) are present in about $5 \%$ at initial stage and in another $5 \%$ during the course of the illness.[17] The most common form is active parenchymal neurocysticercosis, and mostly presents with convulsions.[18] There is generally a good response to antiepileptic drugs. No evidence suggests that occurrence of vertical transmission to the foetus. However, horizontal transmission from the mother to the neonate is noted and therefore screening of mothers with NCC is mandatory.[19] In Most pregnancies, women with NCC had good outcomes and no evidence that maternal NCC causes foetal anomaly or brain damage.[9] However, sustained seizures itself is a high risk factor to the foetus due to hypoxia.[12]

In our study, MRI Brain was s/o neurocysticercosis. The patient was started on anti-convulsant therapy advised by neurologists. Management of neurocysticercosis in pregnancy is mainly aimed to control symptoms with first-line antiepileptic therapy. Antiparasitic agents, albendazole or praziquantel, should be started only after seizures control since the acute inflammation caused by degenerating cysts may exacerbate symptoms. ${ }^{[20]}$ Anti-helminthic may exacerbate the inflammation and cerebral oedema and so deferred till the seizure is control. During pregnancy, Praziquantel is preferred over albendazole since the latter can have a worse teratogenic profile. Prevention of this parasitic infection done by interrupting the life cycle of the parasit 


\section{CONCLUSIONS}

Co-existing neurocysticercosis mimicking eclampsia in a k/c/o pre-eclampsia is one of the rarest presentations. In a case of seizures in pregnancy presenting like eclampsia with slightest doubt of atypical presentation should be assessed by neurologist and obstetricians should consider MRI brain to clinch the diagnosis. This could also avoid early termination of pregnancy which is the treatment of eclampsia.

\section{REFERENCES}

[1] Del Brutto OH, Rajshekhar V, White AC, et al. Proposed diagnostic criteria for neurocystecercosis. Neurology 2001;57(2):177-83.

[2] Garcia $\mathrm{HH}$, Del Brutto $\mathrm{OH}$. Imaging findings in neurocysticercosis. Acta Trop 2003;87(1):71-8.

[3] Saurez VR, Iannucci TA. Neurocysticercosis in pregnancy: a case initially diagnosed as eclampsia. Obstet Gynecol 1999;93(5 Pt 2):816-8.

[4] Richards AM, Moodley J, Graham DI, et al. Active management of the unconscious eclamptic patient. Br J Obstet Gynaecol 1986;93(6):554-62.

[5] Sibai BM, Fairlie FM. Eclampsia. In: Gleicher N. Principles and practice of medical therapy in pregnancy. $2^{\text {nd }}$ edn. New York: Appleton \& Lange 1992: p. 880-8.

[6] Del Brutto OH, Nash TE, White AC Jr, et al. Revised diagnostic criteria for neurocysticercosis. J Neurol Sci 2017;372:202-10.

[7] Garcia HH, Nash TE, Del Brutto OH. Clinical symptoms, diagnosis and of neurocysticercosis. Lancet Neurol 2014;13(12):1202-15.

[8] Webb CM, White AC Jr. Update on the diagnosis and management of neurocysticercosis. Curr Infect Dis Rep 2016;18(12):44.
[9] Webb C, Rosa ML, Olson G, et al. Neurocysticercosis in pregnancy. AJP Rep 2018;8(2):e51-6.

[10] Kurl R, Montella KR. Cysticercosis as a cause of seizure disorder in pregnancy: case report and review of literature. Am J Perinatol 1994;11(6):409-11.

[11] Singhal SR, Nanda S, Singhal SK. Neurocysticercosis as an important differential of seizures in pregnancy: two case reports. J Med Case Reports 2011;5:206.

[12] Gardner E, Chang M, Mancuso P, et al. Neurocysticercosis in pregnancy: not just another headache. Nurs Womens Health 2012;16(2):118-24.

[13] Sahai S, Sahai A. Pica causing neurocysticercosis in pregnancy presenting as eclampsia: a report of two cases. J Obstet Gynaecol India 2013;63(1):68-9.

[14] Suarez VR, Iannucci TA. Neurocysticercosis in pregnancy: a case initially diagnosed as eclampsia. Obstet Gynecol 1999;93(5 Pt 2):816-8.

[15] Winkler AS. Neurocysticercosis in sub-Saharan Africa: a review of prevalence, clinical characteristics, diagnosis and management. Pathog Glob Health 2012;106(5):26174.

[16] Ndimubanzi PC, Carabin H, Budke CM, et al. A systematic review of frequency of neurocysticercosis with a focus on people with epilepsy. PLoS Negl Trop Dis 2010;4(11):e870.

[17] Shandera WX, White AC Jr, Chen JC, et al. Neurocysticercosis in Houston, Texas. A report of 112 cases. Medicine (Baltimore) 1994;73(1):37-52.

[18] White AC Jr. Neurocysticercosis: a major cause of neurological disease worldwide. Clin Infect Dis 1997;24(2):101-15.

[19] Asnis D, Kazakov J, Toronjadze T, et al. Neurocysticercosis in the infant of a pregnant mother with a Tapeworm. Am J Trop Med Hyg 2009;81(3):449-51.

[20] Garcia HH, Pretell JE, Gilman RH, et al. A trial of antiparasitic treatment to reduce the rate of seizures due to cerebral cystercercosis. N Engl J Med 2004;350(3):24958. 